\title{
Entanglement Screening by Nonlinear Resonances
}

\author{
Ignacio García-Mata, ${ }^{1, *}$ André R. R. Carvalho, ${ }^{2}$ Florian Mintert, ${ }^{3}$ and Andreas Buchleitner ${ }^{4}$ \\ ${ }^{1}$ Laboratoire de Physique Théorique, UMR 5152 du CNRS, Université Paul Sabatier, 31062 Toulouse Cedex 4, France \\ ${ }^{2}$ Department of Physics, Faculty of Science, Australian National University ACT 0200, Australia \\ ${ }^{3}$ Physics Department, Harvard University, Cambridge, Massachusetts 02138, USA \\ ${ }^{4}$ Max-Planck-Institut für Physik komplexer Systeme, Nöthnitzer Str. 38, D-01187 Dresden, Germany
}

(Received 19 December 2006; published 23 March 2007)

\begin{abstract}
We show that nonlinear resonances in a classically mixed phase space allow us to define generic, strongly entangled multipartite quantum states. The robustness of their multipartite entanglement increases with the particle number, i.e., in the semiclassical limit, for those classes of diffusive noise which assist the quantum-classical transition.
\end{abstract}

PACS numbers: 03.67.Pp, 03.65.Yz, 03.67.Mn, 05.45.Mt

The ultimate success of the quantum information and computation program will depend on our theoretical understanding and experimental control of quantum entanglement. In order to compete with the available classical supercomputing resources, a future quantum computer will have to be composed of a large, i.e., at least mesoscopic number of qubits, and their coherence will need to be preserved over a significant period of time. Contemplating the fact that entanglement is a manifestation of multiparticle coherence, and that the density of states explodes exponentially with the particle number, it is easy to appreciate the dimension of the challenge ahead.

So far, little is known about entanglement in open quantum systems - where "open" refers to the unavoidable coupling to uncontrolled degrees of freedom in the "environment," which eventually induces decoherence in the system dynamics. Only recently [1-7] have there been first theoretical and experimental attempts to characterize entanglement dynamics under decoherence, but a sufficiently general picture still has to emerge. In particular, most studies did so far focus on specific classes of highly entangled $W, \mathrm{GHZ}$, or cluster states, and on their specific robustness against certain sources of decoherence $[8,9]$. As the number of particles increases, the faithful experimental generation and probing of these states tends to become more and more difficult, with rapidly increasing experimental overhead. Furthermore, whether and in which sense their entanglement properties can be considered as "generic" is a largely open issue, given the complicated topology of state space.

In our present contribution, we will adopt a different perspective, which imports some generic features of quantum dynamics with underlying mixed, regular-chaotic phase space structure, i.e., from quantum chaos [10]. In contrast to earlier studies of the impact of mixed phase space dynamics [11-13] and nonlinear light-matter interaction $[14,15]$ on bipartite entanglement, we are here interested in the multipartite limit of large particle numbers. This is of crucial importance in the context of entanglement scaling alluded to above and will be identified with the semiclassical limit of progressively finer (quantum) resolution of classical phase space structures, by a suitable definition of many-particle basis states in terms of classical phase space coordinates. We will see that nonlinear resonances, which are ubiquitous in classical Hamiltonian systems [16], naturally define strongly — though not maximally - entangled multipartite quantum states. This nonoptimality is compensated by the nonlinear resonance structure providing a natural shelter against certain types of decoherence: Indeed, the robustness of the associated multipartite entangled states is found to increase with the number of particles, i.e., in the semiclassical limit.

We start out with a system of $k$ qubits that lives on a Hilbert space with tensor structure $\mathcal{H}=\mathcal{H}_{1} \otimes$ $\mathcal{H}_{2} \otimes \cdots \otimes \mathcal{H}_{k}$, where each factor space $\mathcal{H}_{j}$ has dimension two. The "computational basis" $\{|i\rangle\}$, which spans $\mathcal{H}$, is given by the $k$-particle product states (i.e., by binary $k$-strings, which we identify with the binary representation of $i=0 \ldots N-1$ ), and has dimension $N=2^{k}$. Maps on classical phase space can be implemented efficiently in such systems after suitable identification of the computational with the position basis $\left\{\left|q_{i}\right\rangle\right\}$, upon the substitution $q_{i}=i / N$ [17-19]. Then, the corresponding momentum basis $\left\{\left|p_{i}\right\rangle\right\}$ is given by the discrete Fourier transform of $\left\{\left|q_{i}\right\rangle\right\}$ [20]. Thus, the phase space is wrapped on a torus, with a phase space area $1 / N$ occupied by a single position basis state. Coherent (or minimum uncertainty) states are obtained as Gaussian wave packets of width $1 / \sqrt{N}$ both in position and momentum [21]. Accordingly, the effective Planck constant is given by $\hbar_{\text {eff }}=1 / 2 \pi N$, and the semiclassical limit $\hbar_{\text {eff }} \rightarrow 0$ of arbitrary phase space resolution by a single quantum state is approached as the particle number $k$ tends to infinity.

Given this quantum coarse graining of phase space, we will now monitor the time evolution of the multipartite entanglement of a quantum state initially prepared as a minimum uncertainty Gaussian wave packet, launched at different positions on the torus (which we will unfold as a unit square, for the ease of illustration). We propagate the wave packet by the unitary operator 


$$
U^{t}=\left(e^{i N \chi_{2} \cos (2 \pi \hat{q})} e^{i N \chi_{1} \cos (2 \pi \hat{p})}\right)^{t},
$$

(with $t$ integer) which follows from the quantization of the classical Harper map

$$
\begin{aligned}
& p^{\prime}=p-\chi_{1} \sin (2 \pi q) \\
& q^{\prime}=q+\chi_{2} \sin \left(2 \pi p^{\prime}\right)
\end{aligned} \quad(\bmod 1) .
$$

Equation (2) describes a particle subject to a periodic impulse, with a position-dependent amplitude [22], and exhibits a transition from regular to chaotic dynamics as the kicking strengths $\chi_{1}, \chi_{2}$ are tuned from $\chi_{1}, \chi_{2} \lesssim 0.11$ to $\chi_{1}, \chi_{2} \gtrsim 0.63$. Here, we employ the fixed values $\chi_{1}=$ $\chi_{2}=\chi=0.4964$, what defines a mixed phase space structure as depicted in the inset of Fig. 1.

The quantum evolution (1) modifies the decomposition of the evolved state $\left|\psi_{t}\right\rangle$ in the computational basis, and thus the entanglement of the $k$ degrees of freedom defined by the constituent qubits. In order to assess the nonclassical correlations inscribed into the evolved state, we use the $k$-partite concurrence $C_{k}$ as defined in [1]. On pure states, this quantity is given by the square root of a balanced average over the squared concurrence of all nontrivial

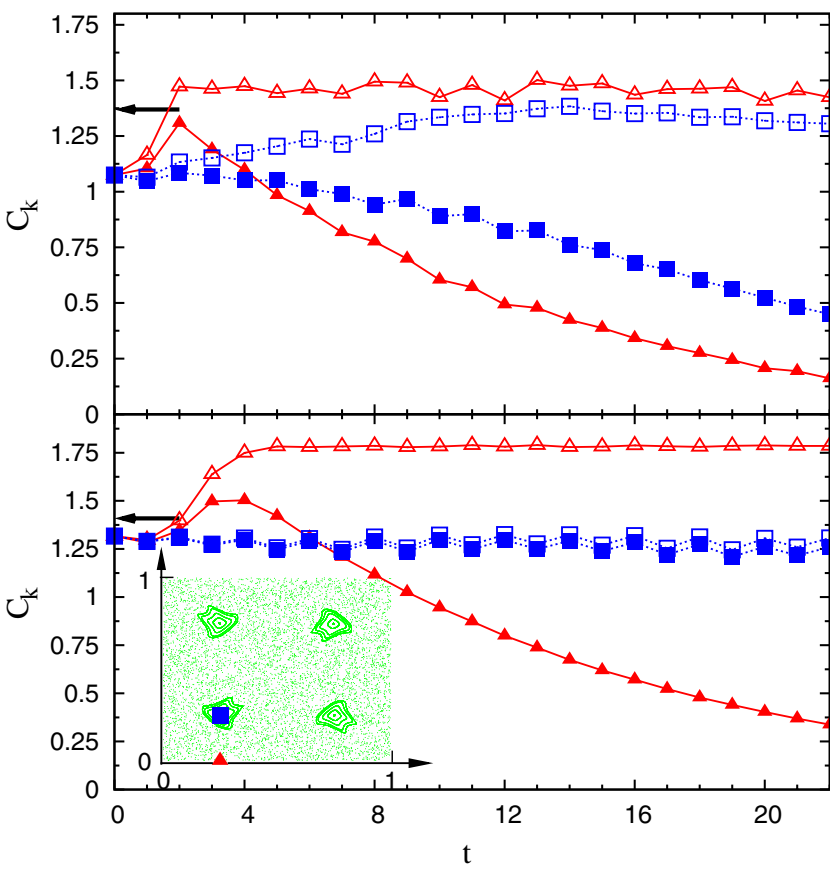

FIG. 1 (color online). Evolution of multipartite concurrence $C_{k}$ for two different numbers of qubits: $k=5$ (top), $k=8$ (bottom). Open symbols refer to unitary dynamics, while filled symbols represent the evolution under the diffusive noise described by Eq. (3). Squares correspond to an initial condition inside the nonlinear resonance island, triangles to initial conditions within the chaotic sea, in the classical phase space spanned by $p$ and $q$ (see inset). For different qubit numbers, the ratio of noise strength to the effective size of Planck's quantum, $\epsilon / \hbar_{\text {eff }}$, is kept constant $(\epsilon=0.04$ for $k=5$, and $\epsilon=$ 0.005 for $k=8$ ). Black arrows indicate the value of $C_{k}$ for $k$-partite GHZ states. bipartitions of the $k$-set under scrutiny, and vanishes exclusively for $k$-separable states [1,23]. Moreover, it has the particularly advantageous property $C_{k}\left(|\phi\rangle \otimes|\psi\rangle_{k-1}\right)=$ $C_{k-1}\left(|\psi\rangle_{k-1}\right), \quad \forall|\phi\rangle \in \mathcal{H}_{1},|\psi\rangle_{k-1} \in \mathcal{H}_{2} \otimes \ldots \otimes \mathcal{H}_{k}$. The latter allows us to compare the entanglement inscribed into quantum states composed of an increasing number of subsystems. Furthermore, $C_{k}$ has a generalization for mixed states (through the convex roof construction [1]), which we will make use of below.

We start out with a short inspection of the entanglement dynamics under purely coherent dynamics, to set the scene. The evolution is generated by application of $U^{t}$, where the integer $t$ counts the number of applications and defines a discrete time. The open symbols in Fig. 1 represent $C_{k}(t)$, for two different initial positions (also indicated by filled triangles and squares in the inset) of the initial minimum uncertainty state in phase space-either within an elliptic island ( $q=p=0.25$; open squares) or within the chaotic phase space component $(q=0.25, p=0.0$; open triangles). Since the computational basis is encoded in position states, both initial conditions define the same initial value $C_{k}(0)\left(C_{5}(0)=1.074386\right.$ and $C_{8}(0)=1.316826$, respectively) of concurrence. Since, on the quantum level, chaotic dynamics is tantamount of strong coupling in any basis, it immediately follows that $C_{k}$ will increase rapidly for the initial condition placed in the chaotic domain and saturate once equilibrated over the chaotic eigenstates [1113] of the quantized Harper map - this is indeed observed in the figure. Note, however, that the saturation level does not coincide with the maximal possible value of $C_{k}$ on the pure states, but rather with its most probable value (which approaches the maximal value in the limit $k \rightarrow \infty$ [24]). Also note that, for $k>3$, this value is larger than the $k$-partite entanglement of GHZ states, which are maximally entangled only in the special case $k=3$ [25]. Indeed, $C_{k=5}^{\mathrm{GHZ}}=1.369286$, and $C_{k=8}^{\mathrm{GHZ}}=1.408798$ (indicated by black arrows in Fig. 1), approaching our minimal uncertainty state's initial multipartite entanglement with increasing $k$. Finally, the qualitative behavior of $C_{k}(t)$ for an initial state lying in the chaotic sea depends only weakly on the number of qubits, as evident from a comparison of both panels in Fig. 1: For $k=8$ (corresponding to a Hilbert space dimension $N=256$ ), the time evolution is very smooth, while for $k=5$ (Hilbert space dimension $N=$ 32 ), still some fluctuations - essentially a finite size effect-are observed.

For the state initially placed within the elliptic island, size does matter: for $k=5$, the initial coherent state cannot be well accommodated within the elliptic island in phase space (due to the finite size of $\hbar_{\text {eff }}$ ) and exhibits nonnegligible tunneling coupling to its chaotic environment. Consequently, as time proceeds, the coherently evolved state spreads more and more over the chaotic phase space component, and its entanglement finally reaches essentially the same value as for the initial condition within 
the chaotic domain, just after considerably longer timeessentially determined by the relevant tunneling matrix elements (which, in general, will be strongly fluctuating under small parameter changes [26,27]). In contrast, for $k=8$, tunneling from the island into the chaotic sea occurs on a much longer time scale (which, on average, increases exponentially with $\hbar_{\text {eff }}$ ) and remains invisible on the time scale covered in Fig. 1. The small oscillations of $C_{k=8}(t)$ are due to the spreading of the initial wave packet along the regular island's tori.

This screening of the initial state from the chaotic sea when initially placed within the elliptic island, more and more efficient with increasing particle number, has an immediate consequence for the robustness of the state's multipartite entanglement under the influence of decoherence, as illustrated by the filled symbols in Fig. 1: For chaotic initial conditions, an initial rise of $C_{k}$ is rather quickly overruled by the loss of multiparticle coherence and hence of entanglement, and this is once again largely independent of $k$. However, for initial conditions within the island, $k=5$ again leads to asymptotically the same behavior as for the chaotic initial condition, while $k=8$ induces entanglement dynamics almost completely unaffected by the noise. Thus, for sufficiently large $k$, equivalent to sufficiently small $\hbar_{\text {eff }}$, and correspondingly suppressed tunneling rates, the classical nonlinear resonance creates strongly entangled multipartite states which, in addition, are robust against noise. This is further illustrated in Fig. 2, where $C_{k=8}(t=16)$ is plotted for different initial conditions, in the absence and in the presence of noise: Clearly, entanglement is robust when shielded by the resonance island. While chaotic dynamics produce slightly stronger entanglement, this is significantly more fragile under decoherence.

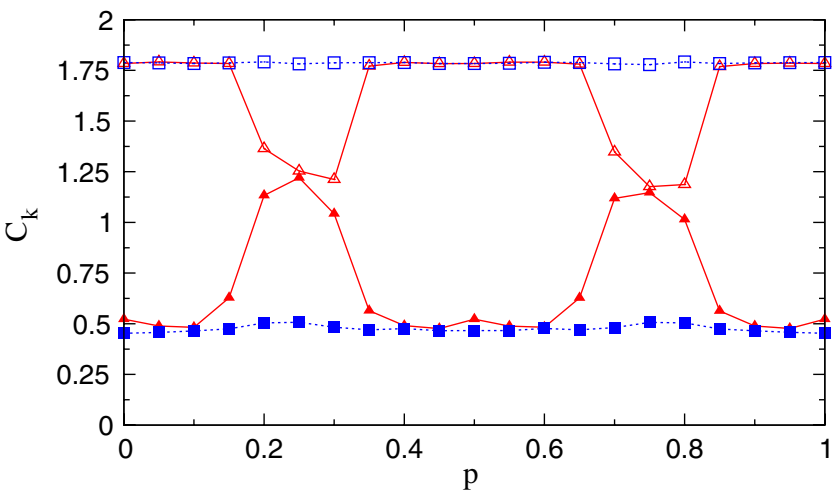

FIG. 2 (color online). Eight-partite concurrence $C_{k=8}$ as a function of initial momentum $(p=0.0,0.05, \ldots, 0.95,1.0)$, after 16 iterations of the Harper map, with $\chi=0.4964$. Open symbols correspond to unitary evolution, while filled symbols refer to unitary evolution amended by diffusive noise, Eq. (3). Squares represent the initial position $q=0.5$, and triangles $q=0.25$. Peaks and dips of $C_{k=8}(t)$ are located exactly at the center of the nonlinear resonance island in classical phase space.
Given the above, some remarks on the applied noise are in order, since any statement on the robustness of some kind of coherence must depend on the nature of the noise. Here, we used Gaussian noise as described in [28,29], which can be implemented experimentally by an enlargement of the quantum register by a set of suitably initialized ancilla qubits [30]. It can be written as a map

$$
\mathbf{S}_{\epsilon}(\rho)=\sum_{q p} c_{\epsilon}(p, q) T_{q p} \rho T_{q p}^{\dagger},
$$

which is applied once after each application of $U^{1} . c_{\epsilon}(p, q)$ is the discrete Fourier transform of $\tilde{c}_{\epsilon}(\mu, \nu)=\exp \left[-\frac{1}{2} \times\right.$ $\left.\left(\frac{\epsilon N}{\pi}\right)^{2}\left(\sin ^{2}[\pi \mu / N]+\sin ^{2}[\pi \nu / N]\right)\right]$ and is very close to a periodic Gaussian of width $\epsilon /(2 \pi)$, centered around $(q, p)=(0,0)$. The $T_{q p}$ are unitary translation operators on the torus [31].

The action of $\mathbf{S}_{\epsilon}(\rho)$ is easily understood in phase space: with high probability, the state is left untouched, while with weight $c_{\epsilon}(p, q)$, locally in phase space, every possible translation is generated. This noise is similar to a high temperature bath of oscillators producing both diffusion and decoherence, and steers the quantum dynamics into the semiclassical limit [32]. In Wigner phase space representation, diffusion induces broadening and blurring of the contour of the state, while decoherence wipes out the interference fringes, and eventually transforms the state into a mixed (classical) state. Given the $k$-dependence of $\hbar_{\text {eff }}$, the strength $\epsilon$ of the noise was scaled such as to keep $\epsilon / \hbar_{\text {eff }}$ constant, in the above plots. The observed robustness of entanglement stems from the local action of $\mathbf{S}_{\epsilon}(\rho)$, which respects the classical phase space structure and leaves the initial state within the elliptic island effectively as a fixed point of the evolution, in the limit of large $k$ (small $\hbar_{\text {eff }}$ ). The dramatically different dynamics of $C_{k=8}$ within and outside the island is a (multipartite) manifestation of enhanced decoherence in classically chaotic as opposed to regular systems [33], for this specific type of noise, and suggests the emergence of a subspace which is shielded against disentanglement, in the semiclassical limit.

In contrast, we may choose nonlocal noise sources such as defined by the multipartite phase damping channel (PDC),

$$
\mathbf{S}_{\epsilon}^{\mathrm{PDC}}(\rho)=(1-\epsilon) \rho+\epsilon \sum_{i} \rho_{i i}|i\rangle\langle i|,
$$

or the generalized depolarizing channel (DPC)

$$
\mathbf{S}_{\epsilon}^{\mathrm{PDC}}(\rho)=(1-\epsilon) \rho+\epsilon I .
$$

For a computational basis identified with position states, both these maps can be expressed in terms of translations on the torus [34], though now with equal (rather than Gaussian, see (3)) weight. Thus, they completely obliterate the classical phase space structure, and couple different eigenstates of the quantized Harper map, irrespectively of 


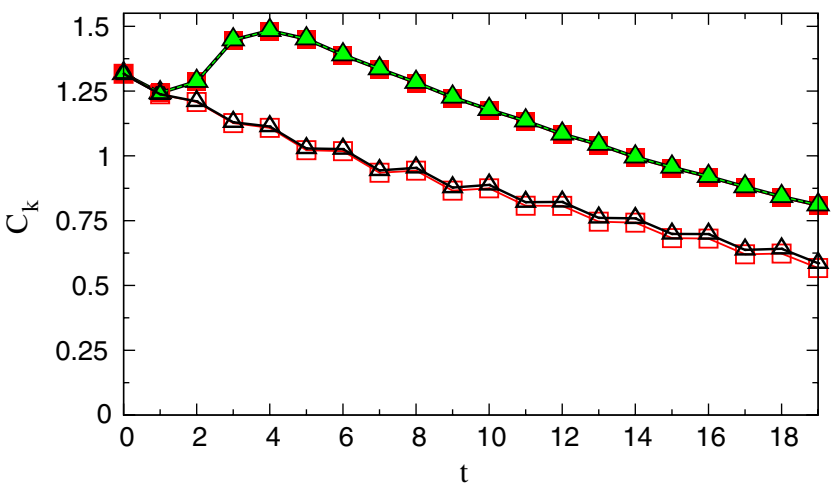

FIG. 3 (color online). Evolution of multipartite concurrence $C_{k=8}$ in the presence of phase damping (triangles, Eq. (4)) and depolarizing noise (squares, Eq. (5)), with $\epsilon=0.04$ in Eqs. (4) and (5). Filled and open symbols refer to initial conditions in the chaotic domain and within the resonance island, respectively, precisely as in Fig. 1.

their localization properties in regular or chaotic phase space domains. Accordingly, given the same initial conditions as in Figs. 1 and 2, concurrence decreases monotonously with time, independently of the initial condition, except for the typical short time transient (see also Fig. 1) observed for the chaotic initial condition - see Fig. 3.

To conclude, we have shown that minimum uncertainty states induce multipartite entanglement in the associated computational basis, robust against the action of diffusive Gaussian noise, when launched within a nonlinear resonance island. They can be produced efficiently as ground states of the Harper Hamiltonian [19], subsequently translated using torus translation operators. The latter generate modular additions with controlled phase shifts on the register qubits and can be implemented, e.g., in ion trap experiments $[35,36]$. Furthermore, given the robust entanglement evolution for initial conditions within a regular island, also those eigenstates of the Harper Hamiltonian which are anchored to the classical regular island exhibit the same robustness properties. Thus, robust multipartite entangled states can be defined through the resonance condition which defines the regular island, a ubiquitous feature of Hamiltonian systems with mixed classical phase space structure.

I. G.-M. was partially supported by CONICET (Argentina) and by the EC IST-FET project EuroSQIP. F. M. acknowledges financial support of Alexander von Humboldt Foundation.

*Electronic address: garcia@irsamc.ups-tlse.fr

[1] F. Mintert, A. R. R. Carvalho, M. Kuś, and A. Buchleitner, Phys. Rep. 415, 207 (2005).
[2] K. Życzkowski et al., Phys. Rev. A 65, 012101 (2001).

[3] B. V. Fine et al., Phys. Rev. B 71, 153105 (2005).

[4] A. R. R. Carvalho et al., quant-ph/0510006.

[5] T. Yu and J.H. Eberly, Phys. Rev. Lett. 97, 140403 (2006).

[6] C. Petitjean and Ph. Jacquod, Phys. Rev. Lett. 97, 194103 (2006).

[7] G. Puentes, D. Voigt, A. Aiello, and J.P. Woerdman, quant-ph/0607014 [Phys. Rev. A (to be published)].

[8] A. R. R. Carvalho, F. Mintert, and A. Buchleitner, Phys. Rev. Lett. 93, 230501 (2004).

[9] W. Dür and H.-J. Briegel, Phys. Rev. Lett. 92, 180403 (2004).

[10] J. Madroñero et al., in Advances in Atomic, Molecular, and Optical Physics, edited by G. Rempe and M. O. Scully (Elsevier, Amsterdam, 2006).

[11] K. Furuya, M. C. Nemes, and G. Q. Pellegrino, Phys. Rev. Lett. 80, 5524 (1998).

[12] P. A. Miller and S. Sarkar, Phys. Rev. E 60, 1542 (1999).

[13] H. Fujisaki et al., Phys. Rev. E 67, 066201 (2003).

[14] A. Kowalewska-Kudlaszyk and W. Leoński, Phys. Rev. A 73, 042318 (2006).

[15] M. K. Olsen, Phys. Rev. A 73, 053806 (2006).

[16] A. J. Lichtenberg and M.A. Lieberman, Regular and Chaotic Dynamics (Springer-Verlag, New York, 1991).

[17] A. J. Scott and C. M. Caves, J. Phys. A 36, 9553 (2003).

[18] C. Miquel, J. P. Paz, and M. Saraceno, Phys. Rev. A 65, 062309 (2002).

[19] J. P. Paz, A. J. Roncaglia, and M. Saraceno, Phys. Rev. A 69, 032312 (2004).

[20] J. H. Hannay and M. V. Berry, Physica D (Amsterdam) 1, 267 (1980).

[21] S. Nonnemacher, Nonlinearity 10, 1569 (1997).

[22] P. Leboeuf, J. Kurchan, M. Feingold, and D. P. Arovas, Phys. Rev. Lett. 65, 3076 (1990).

[23] R. Demkowicz-Dobrzański et al., Phys. Rev. A 74, 052303 (2006).

[24] C. Viviescas et al. (to be published).

[25] This corrects an earlier statement in [8].

[26] S. Tomsovic and D. Ullmo, Phys. Rev. E 50, 145 (1994).

[27] C. Eltschka and P. Schlagheck, Phys. Rev. Lett. 94, 014101 (2005).

[28] S. Nonnenmacher, Nonlinearity 16, 1685 (2003).

[29] I. García-Mata and M. Saraceno, Phys. Rev. E 69, 056211 (2004).

[30] I. García-Mata (unpublished).

[31] J. Schwinger, Proc. Natl. Acad. Sci. U.S.A. 46, 570 (1960).

[32] A.R. R. Carvalho et al., Phys. Rev. E 70, 026211 (2004).

[33] W. H. Zurek and J.P. Paz, Phys. Rev. Lett. 72, 2508 (1994).

[34] M. L. Aolita et al., Phys. Rev. A 70, 062301 (2004).

[35] V. Vedral et al., Phys. Rev. A 54, 147 (1996).

[36] C. Miquel et al., Phys. Rev. A 54, 2605 (1996). 